\title{
Evaluation of the activity and medical nutrition therapy types used by nutrition support teams in hospitals in the Greater Poland voivodeship
}

\author{
Paweł Juszczak ${ }^{1,2}$, Jakub Noskiewicz' ${ }^{1}$ Patrycja Sosnowska², Michał Sawicki², Magdalena Frankowicz ${ }^{2}$, \\ Jarosław Rogacki², Przemysław Mańkowski² \\ ${ }^{1}$ Department of Human Nutrition and Dietetics, Poznan University of Life Sciences, Poznan, Poland \\ ${ }^{2}$ Department of Paediatric Surgery, Traumatology, and Urology, Poznan University of Medical Sciences, Poznan, Poland
}

Gastroenterology Rev 2021; 16 (1): 43-46

DOI: https://doi.org/10.5114/pg.2021.104735

Key words: nutrition support team, nutrition therapy, enteral nutrition, parenteral nutrition.

Address for correspondence: Jakub Noskiewicz PhD, Institute of Human Nutrition and Dietetics, Poznan University of Life Sciences, Poznan, Poland, phone: +48 609355 609, e-mail: jnoskiewicz@op.pl

\begin{abstract}
Introduction: Nutrition therapy is medical treatment that includes assessment of patients' nutritional status, their nutrient requirements, supply of nutritional products in appropriate quantities, and monitoring all of these processes. The nutrition support team is responsible for the organization of the nutrition therapy.

Aim: To evaluate the activity of nutrition support teams in hospitals in the Greater Poland voivodeship.

Material and methods: An evaluation of nutrition support teams' activity and the types of nutrition therapy used in hospitals in the Greater Poland voivodeship was made. In order to collect all the necessary data, a questionnaire with closed-ended questions was sent to all hospitals registered in the voivodeship.

Results: Out of 25 hospitals that returned the completed questionnaire, 15 (60.0\%) confirmed the presence of a nutrition support team in their structures. In previous reports it was 17 centres (68.0\%) that had such team, but sometimes the team was not working to its full extent. $73.0 \%$ of hospitals were satisfied with both the presence of the team and its activity concerning proper fulfilment of entrusted tasks. The activity of nutrition support teams in the Greater Poland voivodeship correlated closely with the size of the hospital.

Conclusions: Nutrition support teams function in the majority of hospitals in the Greater Poland voivodeship. Nutrition teams are more common in larger hospitals. In order to provide proper medical nutrition therapy, the legal obligation to appoint nutrition support teams in the hospitals should be restored.
\end{abstract}

\section{Introduction}

Nutrition therapy is medical treatment that includes assessment of patients' nutritional status, their nutrient requirements, supply of nutritional products in appropriate quantities, and the monitoring all of these processes. The nutrition support team is responsible for the organization of nutrition therapy in hospitalized patients. In 2011 the Minister of Health in Poland adopted a law concerning a legal obligation for hospitals to appoint nutrition support teams. Such teams were to consist of at least a doctor, a pharmacist, a dietitian, and a nurse. Each member of the nutrition support team was required to complete a course in parenteral and enteral nutrition. However, the regulation was can- celled less than 2 months later by the same Minister of Health. Nonetheless, despite the lack of legal necessity, many hospitals created such teams that still exist and are responsible for the organization of nutrition therapy within the hospital. The main scope of these teams is as follows: to monitor nutritional treatment, to supervise the monitoring of patients' nutritional status, and to implement all of the necessary procedures to prevent the patients' malnutrition.

\footnotetext{
Aim

The aim of this report is to evaluate the activity of nutrition support teams in hospitals in the Greater Poland voivodeship.
} 


\section{Material and methods}

On the basis of the survey, an evaluation of both nutrition support teams' activity and types of nutrition therapy used in hospitals in the Greater Poland voivodeship was made.

In order to collect all the necessary data, a questionnaire with 10 closed-ended questions (multiple-response questions) was sent to all hospitals registered in the Greater Poland voivodeship in 2018. The questions concerned the state of supervision over ongoing nutrition therapy and methods how it was organized and managed in the hospital. Answers to the questions were obtained anonymously. Out of 45 sent questionnaires, 25 were fully completed and returned (55.6\%). In the majority of cases ( $n=24,96.0 \%)$, the answers were obtained from public hospitals; in 1 case $(n=1$, $4.0 \%$ ) it was from a non-public unit. Health centres located in cities of up to 50,000 inhabitants dominated $(n=18,72.0 \%)$. Data were received from small, medium, and large health care units. More than half of them treated newborns, infants, and children up to the age of 18 years on a daily basis (Figure 1 ).

\section{Statistical analysis}

The obtained data were analysed using the descriptive statistics methods and tools, adopting the significance level of $p<0.05$.

\section{Results}

Out of 25 hospitals that returned the completed questionnaire, 15 (60.0\%) confirmed the presence of a nutrition support team in their structure. In previ-

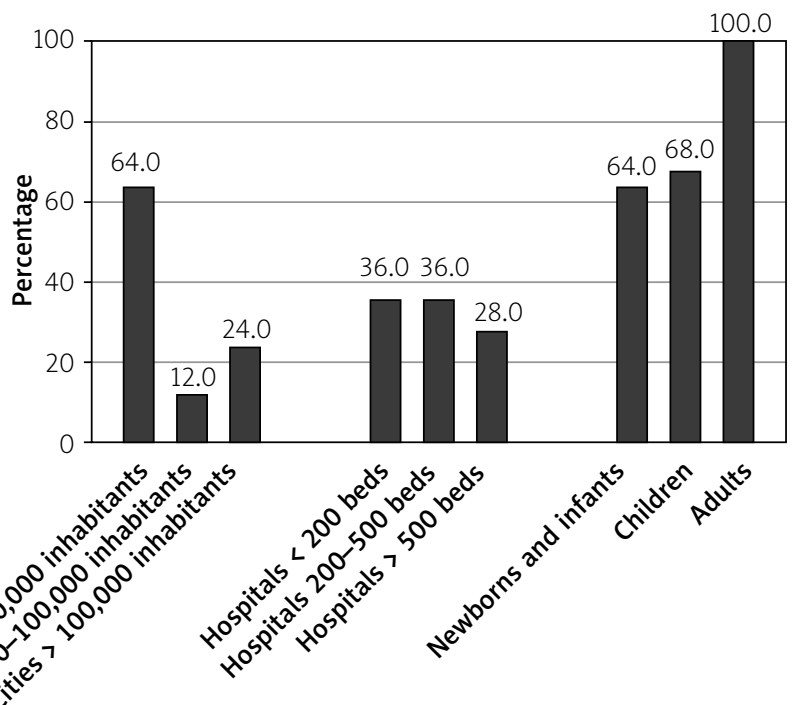

Figure 1. Detailed characteristics of hospitals participating in the study ous reports it was 17 centres (68.0\%) that had such a team, but sometimes it was not working to its full extent. The presence of a functioning nutrition support team was noted in $53.3 \%$ of hospitals treating newborns and infants, while in hospitals for older children this was $70.6 \%$. The medical procedures performed in the group of newborns and infants or children did not increase the probability of organizing the nutrition team ( $p=0.6968$ for patients up to 1 year of age and $p=1.0000$ for older patients). Both past and current activity of nutrition support teams in Greater Poland Voivodeship do correlate closely with the size of a hospital (respectively, $p<0.0001$ and $p=0.0001$ ) - the larger the health care centre, the greater the chance for nutrition support team to functionate (Figure 2). However, $73.0 \%$ of hospitals were satisfied with both the presence of the team and its activity concerning proper fulfilment of entrusted tasks. There was no correlation observed between the proper functioning of the nutrition support team and the size of the hospital ( $p=0.4860$ ) or the presence within the hospital of a parenteral nutrition laboratory $(p=0.1130)$. Nutrition support teams consisted of a doctor, a pharmacist, a dietitian, and a nurse, which met the criteria of ministerial regulation that is no longer valid. No other hospital employees were included in the teams. The presence of a doctor and a pharmacist was declared by all hospitals where proper internal units to monitor nutrition therapy for patients existed, while a nurse and a dietitian were included in over three-quarters of such units only (Figure 3).

Additionally, $33.3 \%$ of hospitals in which nutrition support teams existed reported having their own parenteral nutrition laboratory with the possibility of preparing individually prescribed formulas for parenteral nutrition. One health care unit had the parenteral nutrition prepared in a different hospital. The remaining $60.0 \%$ of centres used ready-to-use 2 - or 3 -chamber

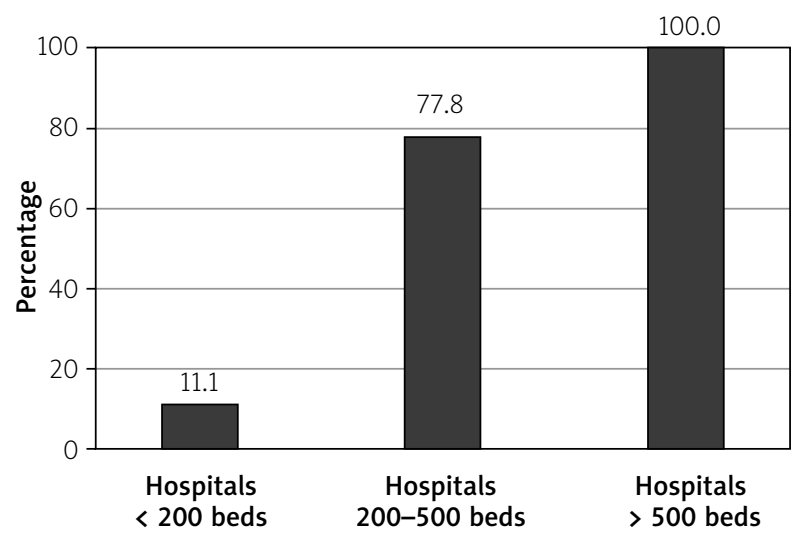

Figure 2. Presence of nutrition support teams depending on the size of the hospital 


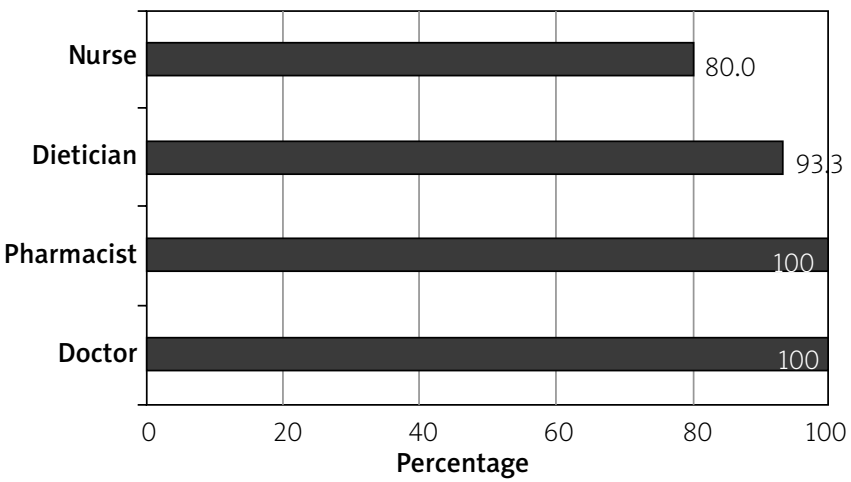

Figure 3. Distribution of the medical professions in nutrition support teams

bags as part of parenteral feeding. Various forms of enteral nutrition were used in the treatment of patients in all hospitals (100.0\%), with oral food supplements (ONS) used in $73.3 \%$ and intravenous feeding in $86.7 \%$ of units (Figure 4).

The presence of the nutrition support team allowed for the analysis of complicated medical cases among them. This procedure enabled team planning of nutritional interventions, especially in patients requiring multidisciplinary treatment and among children. It also reduced the risk of complications related to nutritional treatment. In our material, complications related with total parenteral nutrition (TPN) were noted in $28.6 \%$ of patients treated in centres with nutritional teams. The percentage of such complications in other hospitals was noted at $39.6 \%$. The most common complications were associated just with TPN. They were manifested by refeeding syndrome and metabolic disorders with longterm parenteral nutrition. The second group of complications associated with TPN was a problem with central vascular access. It affected less than $10.0 \%$ of patients. Most often it was associated with catheter mechanical disorders or bacteraemia caused by catheter infections.

\section{Discussion}

The obtained value of the response rate is comparable with the values given in similarly constructed surveys that were conducted in other countries in Europe. For example, Folgueras et al. presented a response rate of $67.0 \%$. At the same time, they noticed that the response rate was higher in lager hospitals, i.e. with more than 200 beds (83.0\%). A similar pattern was observed by the authors of this publication. Nearly two-thirds of hospitals in the Greater Poland voivodeship currently have a nutrition support team responsible for the organization of nutrition therapy. This includes mostly nutritional treatment and malnutrition prevention in hospitalized patients. Despite the fact that the Minister

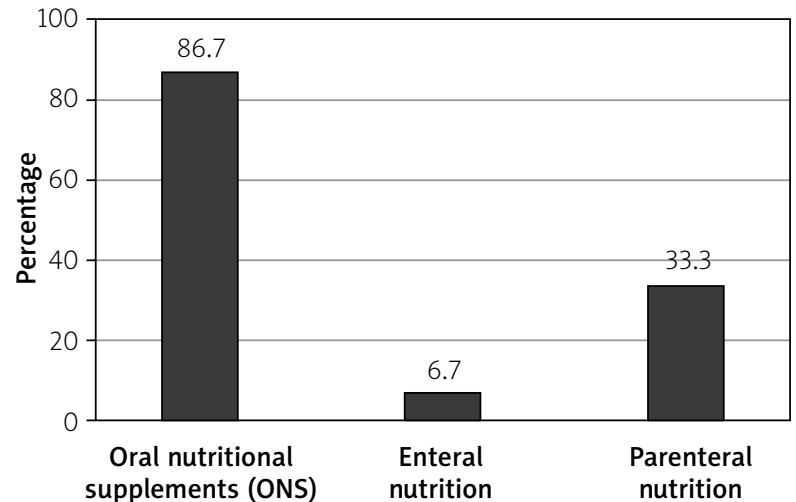

Figure 4. Types of nutritional treatment used in hospitals in the Greater Poland voivodeship

of Health in Poland waived the legal obligation for hospitals to provide a nutrition support team, only in few hospitals teams have they actually been resolved. This may prove the usefulness of nutrition teams and their significant influence on the nutrition therapy of hospitalized patients. It should also be noted that the larger the hospital, the greater the chance of the existence of a proper unit responsible for supervising the nutrition therapy in patients. On the one hand, it is associated with more frequent hospitalization of multidisciplinary patients in these units. These patients require many different specialists in the therapeutic process, including professionals in nutrition therapy. On the other hand, it results from the need for accreditation, which is more often carried out in larger units. At the same time, the size of the hospital or the possibility of conducting advanced nutrition therapy procedures within the hospital (i.e. individually prescribed parenteral nutrition) do not affect the importance or the quality of the work of nutrition teams. These teams function equally well in smaller units. Implementation of nutritional documentation into the patient's medical documentation has a significant impact on the results of the nutrition support team's action. It can easily be introduced in district hospitals, as well. Although it was proven that child patients are among the most vulnerable to malnutrition, it does not correlate with the more frequent setting up of nutrition support teams in paediatric or neonatal hospitals. In this group of patients, malnutrition, especially in emergency cases, is often underestimated and significantly increases both the time of hospitalization and costs of treatment [1-3].

In the studied population the main and also the most important members of the nutrition teams were the doctor taking care of clinical nutrition and the pharmacist from the hospital pharmacy. Less often, but still in the majority of cases, the team was supplemented by a dietitian and a nurse. De Longueville et al. noticed 
that a dietitian is an important, if not the most important, member of the nutrition support team. In Spain it is the dietitian who plays a decisive role in nutrition teams, including the post of team leader. This results from a lack of involvement of doctors in such teams in this country. Folgueras et al. emphasize, however, that nutrition teams that consist of different specialists work better and are more useful. Unfortunately, such teams are formed less frequently. Nutritional teams consist of various specialists who make better use of tools (i.e. computer programs supported by artificial intelligence (AI)) for analysis of the nutrition of patients and for assessment of the use of nutritional interventions. What is more, the tools introduced to support nutritional work are not always economically beneficial [1-5].

Numerous researchers emphasize the significance of the activity of nutrition teams, in both paediatric and adult hospitals. These teams greatly influence the organization of nutritional rehabilitation. Their activity contributes to improvement of the quality of provided medical services, shortening the time of hospitalization, and reducing the costs of treatment. For example, in hospitals with nutrition support teams, the mortality rate after implementation of percutaneous endoscopic gastrostomy (PEG) is lower. Regular observation of patients with gastrostomies carried out by the nutrition team affects the improvement of the care quality without increasing the costs of treatment. However, there are no unambiguous opinions as to the effectiveness of the team dealing exclusively with the control of TPN. It was proven that patients controlled by such teams have reduced incidence of vascular access complications, but unfortunately it does not correlate with a decreased risk of sepsis. A positive impact of the control carried out by teams for parenteral nutrition on the correct supply of energy to patients was noticed, but it is not so obvious in protein, macro-, and microelement supplementation. The organization of a nutrition team that takes care only of parenteral nutrition seems to be an incorrect procedure [2, 6-8].

\section{Conclusions}

Despite the lack of legal necessity, nutrition support teams exist in most of the hospitals in the Greater Poland voivodeship - most often still meeting the requirements of the Minister of Health that are no longer valid. The activities of the nutrition support teams are mostly noticeable in larger hospitals, where they are responsible for both the organization and supervision of nutrition therapy in the hospital. In order to provide proper nutrition therapy, the legal obligation to appoint nutrition support teams in the hospitals should be restored.

\section{Conflict of interest}

The authors declare no conflict of interest.

\section{References}

1. Kokura Y, Wakabayashi H, Maeda K, et al. Impact of a multidisciplinary rehabilitation nutrition team on evaluating sarcopenia, cachexia and practice of rehabilitation nutrition. J Med Invest 2017; 64: 140-5.

2. Folgueras TM, Ballesteros Pomar MD, Burgos Pelaz R, et al. Organization and management of clinical nutrition in Spain. How do we assess the quality of our activities? Nutr Hosp 2017; 34: 989-96.

3. Tanswell I, Barrett D, Emm C, et al. Assessment by a multidisciplinary clinical nutrition team before percutaneous endoscopic gastrostomy placement reduces early postprocedure mortality. JPEN J Parenter Enteral Nutr 2007; 31: 205-11.

4. De Longueville C, Robert M, Debande M, et al. Evaluation of nutritional care of hospitalized children in a tertiary pediatric hospital. Clin Nutr ESPEN 2018; 25: 157-62.

5. Suárez-Llanos J, Vallejo-Torres L, García-Bello M, et al. Cost-effectiveness of the hospital nutrition screening tool CIPA. Arch Med Sci 2020; 16: 273-81.

6. Fernandez R, Griffiths R, Naylor CJ. Effectiveness of a multidisciplinary total parenteral nutrition team in the hospital setting. JBI Libr Syst Rev 2003; 1: 1-45.

7. Rinninella E, Ruggiero A, Maurizi P, et al. Clinical tools to assess nutritional risk and malnutrition in hospitalized children and adolescents. Eur Rev Med Pharmacol Sci 2017; 21: 2690-701.

8. Scott F, Beech R, Smedley F, et al. Prospective, randomized, controlled, single-blind trial of the costs and consequences of systematic nutrition team follow-up over 12 months after percutaneous endoscopic gastrostomy. Nutrition 2005; 21: 1071-7.

Received: 19.12 .2019

Accepted: 24.04 .2020 